Article

\title{
Evaporation Patterns of Dextran-Poly(Ethylene Glycol) Droplets with Changes in Wettability and Compatibility
}

\author{
Chiho Watanabe ${ }^{1, *(\mathbb{C})}$ and Miho Yanagisawa ${ }^{2,3,4}(\mathbb{D}$
}

1 School of Integrated Arts and Sciences, Graduate School of Integrated Sciences for Life, Hiroshima University, Kagamiyama 1-7-1, Higashi-Hiroshima 739-8521, Japan

2 Komaba Institute for Science, Graduate School of Arts and Sciences, The University of Tokyo, Komaba 3-8-1, Tokyo 153-8902, Japan; myanagisawa@g.ecc.u-tokyo.ac.jp

3 Universal Biology Institute, Graduate School of Arts and Sciences, The University of Tokyo, Komaba 3-8-1, Tokyo 153-8902, Japan

4 Department of Physics, Graduate School of Science, The University of Tokyo, Hongo 7-3-1, Tokyo 113-0033, Japan

* Correspondence: cwatan@hiroshima-u.ac.jp

check for updates

Citation: Watanabe, C.; Yanagisawa, M. Evaporation Patterns of DextranPoly(Ethylene Glycol) Droplets with Changes in Wettability and Compatibility. Life 2022, 12, 373. https://doi.org/10.3390/ life12030373

Academic Editor: Lígia R. Rodrigues

Received: 31 January 2022

Accepted: 1 March 2022

Published: 4 March 2022

Publisher's Note: MDPI stays neutral with regard to jurisdictional claims in published maps and institutional affiliations.

Copyright: (C) 2022 by the authors. Licensee MDPI, Basel, Switzerland. This article is an open access article distributed under the terms and conditions of the Creative Commons Attribution (CC BY) license (https:// creativecommons.org/licenses/by/ $4.0 /)$.

\begin{abstract}
The dextran-PEG system is one of the most famous systems exhibiting phase separation. Various phase behaviors, including the evaporation process of the dextran-PEG system, have been studied in order to understand the physicochemical mechanism of intracellular phase separation and the effect of condensation on the origin of life. However, there have been few studies in dilute regime. In this study, we focused on such regimes and analyzed the pattern formation by evaporation. The specificity of this regime is the slow onset of phase separation due to low initial concentration, and the separated phases can have contrasting wettability to the substrate as evaporation progresses. When the polymer concentration is rather low $(<5 \mathrm{wt} \%)$, the dextran-PEG droplets form a phaseseparated pattern, consisting of PEG at the center and dextran ring of multiple strings pulling from the ring. This pattern formation is explained from the difference in wettability and compatibility between dextran and PEG upon condensation. At the initial dilute stage, the dextran-rich phase with higher wettability accumulates at the contact line of the droplet to form a ring pattern, and then forms multiple domains due to density fluctuation. The less wettable PEG phase recedes and pulls the dextran domains, causing them to deform into strings. Further condensation leads to phase separation, and the condensed PEG with improved wettability stops receding and prevents a formed circular pattern. These findings suggest that evaporation patterns of polymer blend droplets can be manipulated through changes in wettability and compatibility between polymers due to condensation, thus providing the basis to explore origins of life that are unique to the process of condensate formation from dilute systems.
\end{abstract}

Keywords: evaporation pattern; dextran; PEG; phase separation; droplets

\section{Introduction}

Evaporation patterns and its condensation process of colloidal suspensions on a substrate have been intensively investigated as fundamental studies for industrial applications, such as ink and printing technologies, as well as daily life phenomena, such as coffee ring pattern, i.e., a circular ring found in coffee stains [1-3]. These patterns are determined by the local balance between the Marangoni effect and capillary force through the modification of surface tension and viscosity [3,4]. Additionally, when phase separation (or density variation) is caused by condensation, the evaporation pattern becomes more diverse. Such an evaporation pattern with phase separation is reported as a simple blood-based diagnostic method [5-7] as well as a medicine quality control [8].

Recently, evaporation of biomolecular droplets has attracted attention as a mechanism for easily condensing biomolecules in relation to "intracellular phase separation" [9-11] 
and "origin of life" [12,13]. For example, the phase separation of dextran and poly (ethylene glycol) (PEG), along with the evaporation process is reported to simultaneously condensate nucleic acids and enhancement of ribozyme activity [13]. In their experimental condition, using dextran (Mw. $10 \mathrm{k}$ ), PEG (Mw. $8 \mathrm{k}$ ), and their total concentration $>2 \mathrm{wt} \%$, the droplet fully adheres to the solid substrate throughout the evaporation process and the droplet shrinks as the evaporation progresses. Given that it is plausible to imagine lowconcentration conditions in origin of life scenarios, the evaporation process from a much lower initial concentration becomes a relevant target for investigation. This is because it is expected to begin with a fairly dilute prebiotic soup. However, evaporation patterns with phase separation have not been reported for very low concentrations of polymer droplets.

In this study, we studied the evaporation patterns of dextran (Mw. $500 \mathrm{k}$ ) and PEG (Mw. $6 \mathrm{k}$ ) aqueous solution in dilute regimes, which also shows phase separation as evaporation progresses. The difference from previous studies lies in kinetic coupling between compatibility and wettability, i.e., the slow onset of phase separation due to low initial concentration and the wettability reversal due to concentration change. At the lower concentration $(<5 \mathrm{wt} \%)$, dextran-rich ring-like deposition occurs at the contact line of the droplet together with phase separation progression. Then, the pinning of the PEG-rich phase is released as evaporation continues to recede the contact line inward of the droplet, dragging the high viscosity dextran-rich phase from the initial contact line. This process creates multiple string-like pattern of dextran-rich phase on the substrate. This pattern is not observed for higher initial concentration solution since the receding of the contact line does not take place due to its high viscosity, nor when the overall viscosity is low, and the entire droplet moves together. This specific string pattern formation was not observed in a previous report using the dextran-PEG system [13], possibly due to their conditions using higher polymer concentrations and a lower molecular weight of dextran. These findings suggest that evaporation patterns of polymer blend droplets can be manipulated through changes in wettability and compatibility between polymers due to condensation. In addition, they will help us to develop a plausible prebiotic model as well as industrial applications, such as self-organizing patterning and body-fluid-based medical diagnostics using a little sample solution.

\section{Materials and Methods}

\subsection{Materials}

Dextran from Leuconostoc mesenteroides, with an average molecular weight of 500,000 $(500 \mathrm{k})$, and fluorescein isothiocyanate-dextran (FITC-Dex $500 \mathrm{k}$ ) were purchased from Sigma-Aldrich (Saint Louis, MO, USA). PEG, with an average molecular weight 6000 $(6 \mathrm{k})$, and glucose were purchased from FUJIFILM Wako Pure Chemical Corporation (Osaka, Japan). Rhodamine B-labeled PEG (RB-PEG) (molecular weight of 5000, 5 k) was purchased from Biochempeg (Watertown, MA, USA). These materials were used without further purification. Milli-Q water (18.2 $\mathrm{M} \Omega \mathrm{cm}$, Merck Millipore, Meguro, Japan) was used for all solutions. Each polymer or glucose solution was prepared as a highconcentration mother solution (10-40 wt\%), and then mixed and/or diluted by water to obtain desired composition and concentration. Rectangular cover slips were purchased from Matsunami Glass Inc., Ltd., Osaka, Japan. All chemicals and materials were used without further purification. The initial concentrations of fluorescent reagents were $0.05 \mathrm{mg} / \mathrm{mL}$ and $0.005 \mathrm{mg} / \mathrm{mL}$ for FITC-Dex and RB-PEG, respectively. Similarly to our previous reports [14,15], the radius of gyration $R_{\mathrm{g}}$ and overlap concentration $c^{*}$ of dextran $500 \mathrm{k}$ and PEG $6 \mathrm{k}$ are estimated and shown in Table 1. 
Table 1. Radius of gyration $R_{\mathrm{g}}$ and approximate overlap concentration $c^{*}$ of PEG $6 \mathrm{k}$ and Dex $500 \mathrm{k}$ estimated as same as [15].

\begin{tabular}{ccc}
\hline & PEG 6 k & Dex 500 k \\
\hline$R_{\mathrm{g}}[\mathrm{nm}]$ & 3.1 & 17 \\
\hline$c^{*}[\mathrm{wt} \%]$ & 8 & 4 \\
\hline
\end{tabular}

\subsection{Sessile Droplet Formation and Its Observation}

Sessile droplets were formed with volume of ca. $0.3 \mu \mathrm{L}$ on a cover glass and dried under ambient temperature $\left(20-24{ }^{\circ} \mathrm{C}\right)$. The relative humidity was not controlled in the experiments. Typically, the relative humilities for summer and winter are $65 \pm 10 \%$ and $30 \pm 10 \%$, respectively. The drying process and/or the final deposition pattern were observed by inverted fluorescence microscope (IX 73, Olympus Corp., Ltd., Tokyo, Japan). The pattern images were taken phase contrast (PH) or fluorescence (FL) by using fluorescent mirror unit (U-FBNA for FITC-Dex and U-FGW for RB-PEG). All images were taken using a color camera (DP74). The microscope, mirror units, and the camera are from Olympus Corp. Image analyses were performed by using a free-software Fiji from the National Institute of Health (USA). The experimental setup is illustrated in Figure 1.

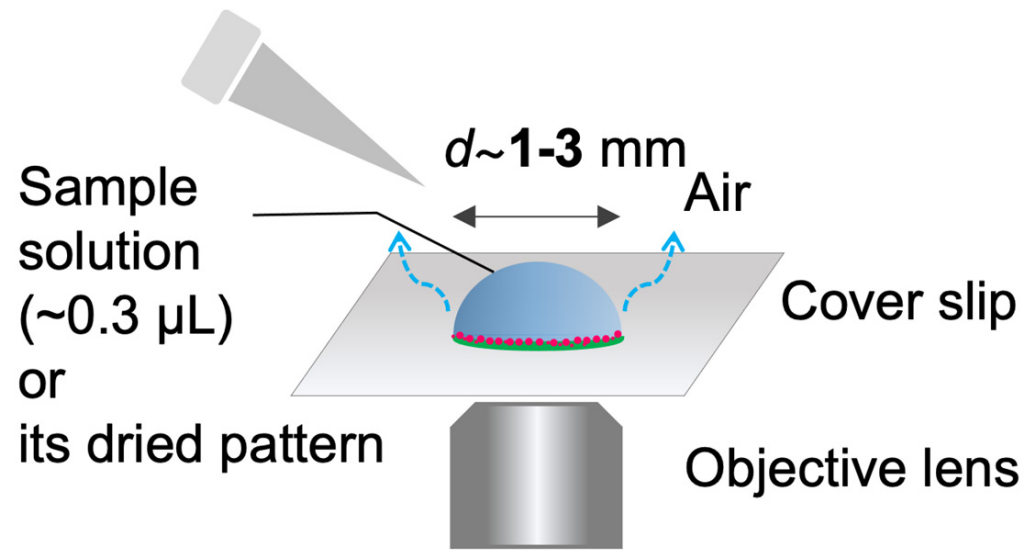

Figure 1. Illustration of the experimental setup.

\subsection{Contact Angle Measurement}

The contact angle was measured by the contact angle meter SImage Entry 6 (Excimer, Inc., Kanagawa, Japan) with the $\theta / 2$ method. The initial contact angle was measured within $10 \mathrm{~s}$ after depositing ca. $2 \mu \mathrm{L}$ solution onto the cover slip. The measurement was repeated at least 3 times to take the average value.

\section{Results and Discussion}

\subsection{Evaporation Patterns for Dextran-PEG Droplets}

We observed the evaporation patterns of binary polymer droplets containing dextran $500 \mathrm{k}$ and PEG $6 \mathrm{k}$. Figure 2a shows a schematic phase diagram of the dextran $500 \mathrm{k}$ and PEG $6 \mathrm{k}$ solutions at room temperature. This dextran-PEG solution is known to exhibit phase separation upon condensation by evaporation. First, the dextran-PEG ratio was fixed to be $4: 1(w / w)$; the initial total polymer concentration $C_{\text {tot }}$ was changed along the red line in Figure 2a. As shown in Figure 2b, the evaporation patterns of the dextran-PEG droplets show change with an increase in $C_{\text {tot }}$. When the $C_{\text {tot }}$ is less than $5 \mathrm{wt} \%$, a ring pattern with multiple strings was observed (Figure $2 \mathrm{~b}$ (top)). The multiple strings extend inward from the outer ring, as illustrated in Figure $2 b$ (top left). Here, we refer to this narrow ring pattern with multiple strings as pattern $A$. As for higher $C_{\text {tot }} \geq 5 \mathrm{wt} \%$, a thicker ring pattern than pattern A was observed (Figure 2c, bottom). Inside the thick ring, there 
are some structures, but not strings. To classify evaporation patterns by the presence or absence of internal strings, we refer to this thick ring pattern without strings as pattern $B$.

(a)

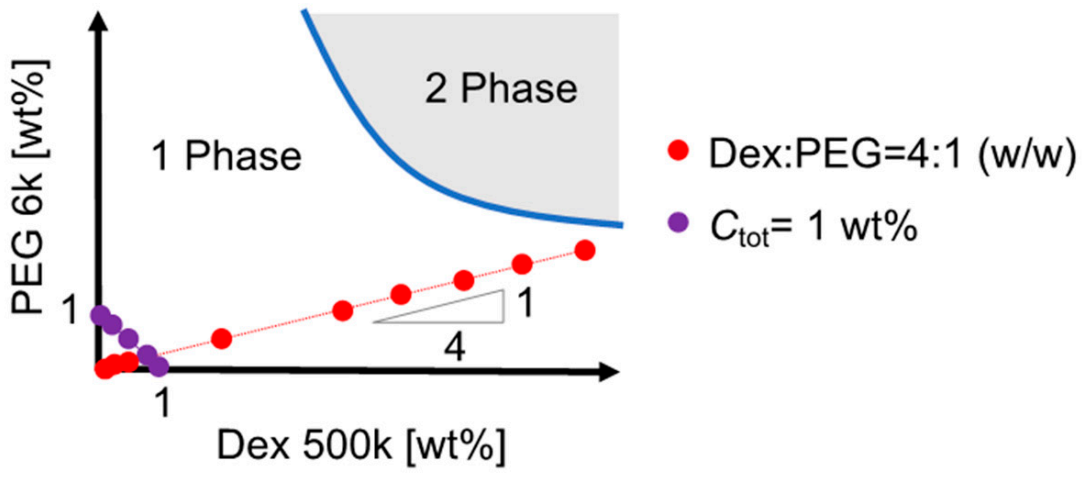

(b) Dex 500k: PEG 6k = 4:1(w/w)

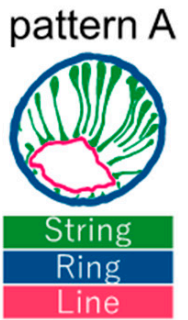

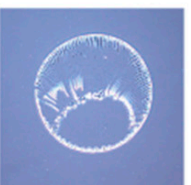

0.1

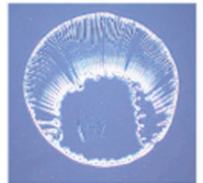

0.25

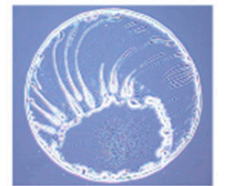

0.5

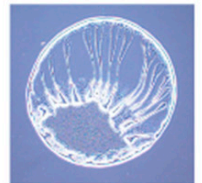

1

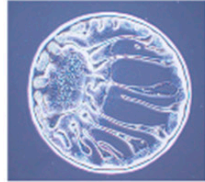

2.5

pattern B

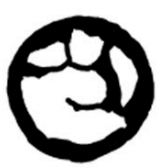

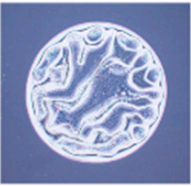

5

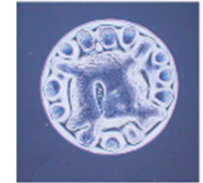

6.25

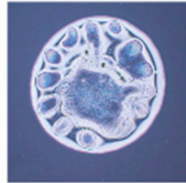

7.5

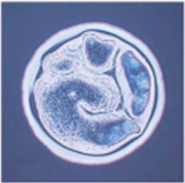

8.75

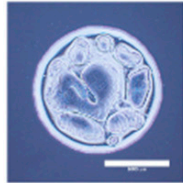

10

Initial concentration $C_{\text {tot }}[\mathrm{wt} \%]$

(c) Initial concentration $C_{\text {tot }}=1 \mathrm{wt} \%$

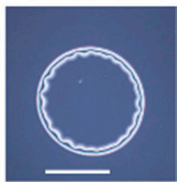

Dex only

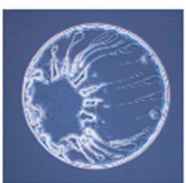

$4: 1$

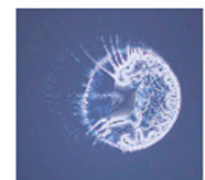

$1: 1$

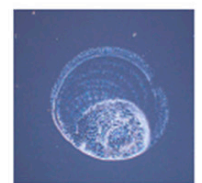

$1: 4$

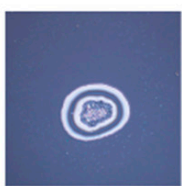

PEG only

Dex: PEG (w/w)

Figure 2. Evaporation patterns for binary polymer droplets of dextran $500 \mathrm{k}$ and PEG $6 \mathrm{k}$. (a) Schematic of the dextran-PEG phase diagram. Red and violet points show the initial concentration and composition of the prepared droplet before the evaporation. The functions of the red and violet points are $y=0.24 x$ and $y=1-x$, respectively. (b) Evaporation patterns of dextran and PEG droplets with different initial polymer concentrations $C_{\text {tot }}$ under a fixed polymer ratio, dextran: PEG $=4: 1$ $(w / w)$. The compositions are indicated with a red line in (a). (c) Evaporation patterns of dextran and PEG droplets with a different polymer ratio, under fixed $C_{\text {tot }}=1 \mathrm{wt} \%$. The compositions are indicated with a violet line in (a). Scale bar: $1 \mathrm{~mm}$.

The ring-like structure common to patterns $\mathrm{A}$ and $\mathrm{B}$ might correspond to the coffee ring pattern upon evaporation. The coffee ring pattern represents a colloid deposition onto a droplet periphery due to colloid-laden droplet evaporation from the contact line, which creates the capillary flow that carries colloids to the contact line from the inside of the droplet [1]. In addition, an unclear internal structure of the pattern B for higher 
$C_{\text {tot }} \geq 5 \mathrm{wt} \%$ is quite similar to the one of previous reports using similar systems (dextran $10 \mathrm{k}$ and PEG $8 \mathrm{k}$ [13]). On the other hand, multiple strings of the pattern A for lower $C_{\text {tot }}<5 \mathrm{wt} \%$ is a novel pattern to the best of our knowledge. Hereafter, we focus on the pattern A with multiple strings inside.

To clarify the conditions under which the pattern A appears, we then fixed the initial polymer concentration $C_{\text {tot }}=1 \mathrm{wt} \%$ and changed the dextran-PEG ratio to be 1:0, 1:4, 1:1, 4:1, and 0:1 $(w / w)$. The examined polymer compositions are indicated as violet points in the phase diagram (Figure 2a). As shown in Figure 2c, the pattern A (i.e., ring pattern with multiple strings) was again observed for the dextran-PEG with a ratio $=4: 1(w / w)$ as shown $C_{\text {tot }}$ to $1 \mathrm{wt} \%$ in Figure $2 \mathrm{c}\left(C_{\text {tot }}<5 \mathrm{wt} \%\right)$. The patterns for dextran: PEG $=4: 1$ and 1:1 look like pattern $\mathrm{A}$; however, the strings extended from the outer ring were less obvious compared to the pattern for dextran: $\mathrm{PEG}=1: 4$.

As for the PEG-only droplet (dextran: PEG = 0:1), a thick ring pattern without strings was observed for all $C_{\text {tot }}$ (Figure 3 (bottom three images)). On the other hand, for the dextran-only droplet (dextran: PEG $=1: 0$ ), an undulated thick ring pattern was observed (Figure 3 (top three images)). This undulation at the contact line of the evaporating droplets was observed for dextran-only droplets, but not for PEG-only droplets. Therefore, the multiple periodic strings of the dextran-PEG droplets (pattern A) might have some relationship with the undulated pattern of the dextran-only droplets. To confirm the idea, we next replace the dextran with glucose which shows no phase separation and lower viscosity.

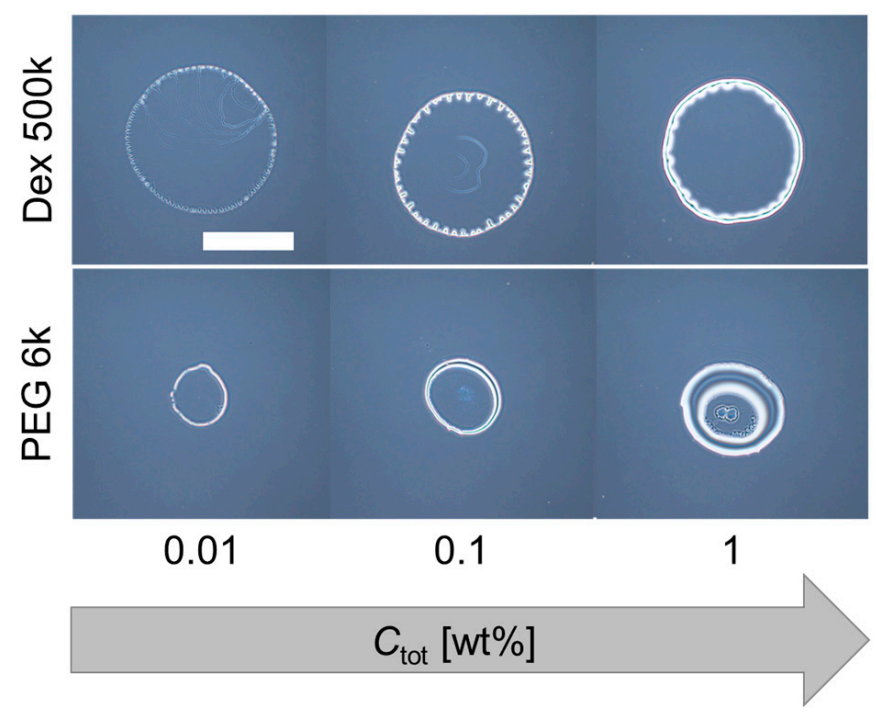

Figure 3. Deposition patterns of dextran only (top) and PEG only (bottom) droplets with different initial concentrations. Scale bar: $1 \mathrm{~mm}$.

\subsection{Evaporation Patterns for Glucose-PEG Droplets}

To verify whether dextran is the origin of the multiple strings in pattern $\mathrm{A}$, dextran was replaced by glucose, the monomer unit of dextran (Figure 4). First, we changed the initial polymer concentration $C_{\text {tot }}$ under a constant polymer ratio, i.e., glucose: PEG $=4: 1$ $(w / w)$. For any $C_{\text {tot }}$ examined, thick ring patterns without internal structure were observed (Figure 4a). Next, we fixed $C_{\text {tot }}=1 \mathrm{wt} \%$ which corresponds to the $C_{\text {tot }}$ of the dextran and PEG system, showing pattern $B$ with multiple stripes (Figure $2 b$, upper), and then varied the glucose-PEG ratio. Similarly, there were no string patterns; however, thick ring patterns without an internal structure or deposit on the center of the droplet were observed at any glucose-PEG ratio (Figure 4 b). These results strongly support our idea that the coexisting condition of large dextran and PEG is a key to obtain the pattern A, i.e., multiple strings extend inward from the outer ring. 
(a) Glucose : PEG6k = $4: 1(w / w)$

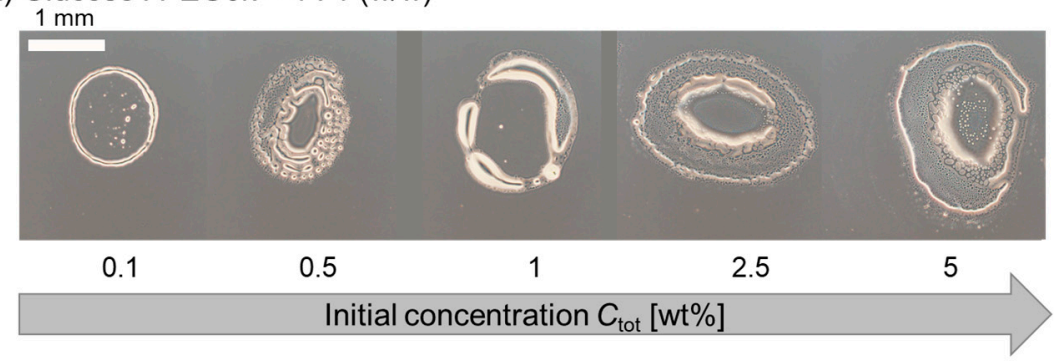

(b) Initial concentration $C_{\text {tot }}=1 \mathrm{wt} \%$

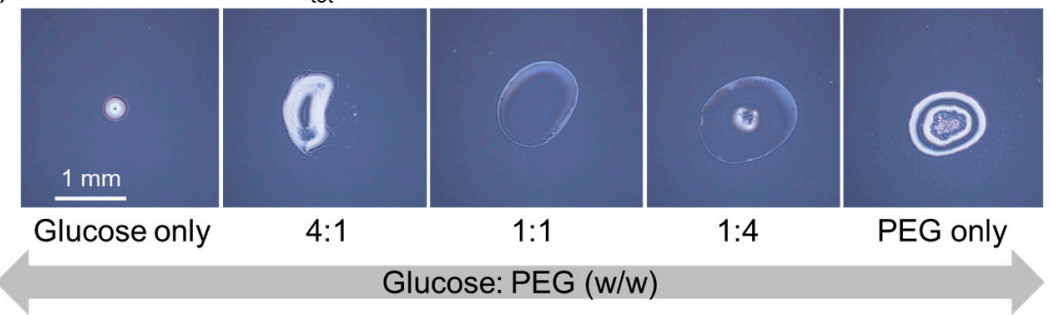

Figure 4. Evaporation patterns for droplets of glucose and PEG solutions. (a) Constant polymer ratio, glucose: PEG $=4: 1(w / w)$ with different initial polymer concentrations $C_{\text {tot }} .(\mathbf{b})$ Constant $C_{\text {tot }}=1 \mathrm{wt} \%$ with a different polymer ratio, glucose-PEG.

\subsection{Fluctuation of Polymer Concentration in Evaporating Dextran-PEG Droplets}

From these results, pattern A (ring pattern with multiple strings inside) is found to be characteristic evaporation pattern for dextran and PEG droplets with a low $C_{\text {tot }}<5 \mathrm{wt} \%$. In addition, undulation of the dextran at the contact line seems to be related to the presence of multiple strings extended from the outer ring. To highlight the relationship between the dextran and string pattern, we visualize the concentration fluctuation upon the evaporation by using FITC-labeled dextran and RB-labeled PEG.

The evaporation processes for (a) dextran-only droplet with $C_{\text {tot }}=0.1 \mathrm{wt} \%$ and (b) PEGonly droplet with $C_{\text {tot }}=0.1 \mathrm{wt} \%$ are shown in Figure 5. For the dextran-only droplet, the ring pattern appears within $50 \mathrm{~s}$ (Figure 5a). The dextran-rich ring at the periphery of the droplet and transits to periodic domains upon the concentration fluctuation. From the phase contrast $(\mathrm{PH})$ image taken after $\sim 265 \mathrm{~s}$, the second and third ring pattern inside are shown (Figure $5 \mathrm{a}$ (far right)). On the contrary, the PEG-only droplet recedes as time evolves, leaving a thick ring-like deposition without any internal structure (Figure $5 b$ ). The ring pattern appeared after $\sim 166 \mathrm{~s}$, which is slower than dextran. We can see that the ring becomes thicker as the PEG polymers at the droplet center move to the ring. In fact, there are no pattern inside the ring, as shown in the PH image (Figure $4 \mathrm{~b}$, right edge). This phenomenon is similar to that of the coffee ring pattern. To quantify the time development of the dextran concentration along the droplet periphery, we analyze the fluorescence intensity of FITC-dextran with time along the cross-sectional plane (dotted line in Figure 5c). Figure 5c (left) shows that the distances between the dextran-rich domains shortened over time, and the shape of the domains elongated toward the droplet center. On the other hand, the outer position of the dextran-rich domain did not change with time and was found to be almost at the same position after $260 \mathrm{~s}$. This is in contrast to PEG in Figure $5 c$ (right), where the outer position of the droplet moved significantly with time.

Finally, the time evolution of the evaporation pattern for dextran and the PEG droplet with $C_{\text {tot }}=0.1 \mathrm{wt} \%$ and the polymer ratio dextran: PEG $=4: 1(w / w)$ (Figures 6 and 7$)$. The whole droplet images and the zoomed images clearly show that dextran firstly condensates at the droplet periphery at $78 \mathrm{~s}$ and the dextran-rich ring pattern transits to periodic domains. This pattern transition is quite similar to the one of dextran-only droplet (Figure 5a). After $78 \mathrm{~s}$, as the contact line of the PEG-rich phase receded, and the periodic dextran-rich domains were pulled toward the droplet center and transited into multiple strings. This formation mechanism of pattern A with a macroscopic flow from outer to inner is different 
from "viscous fingering" where a high viscosity fluid is extruded into a low viscosity fluid [16].

(a) $0.1 \mathrm{wt} \%$ Dextran with FITC-Dex500k

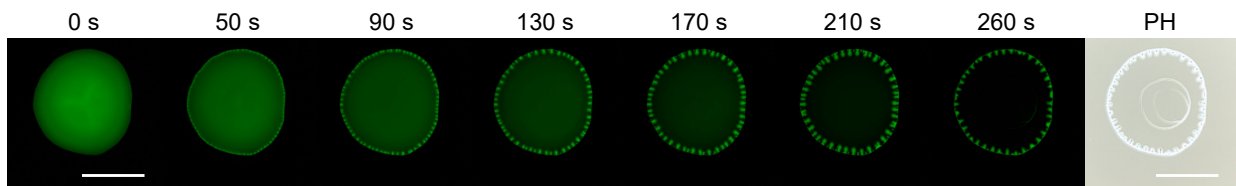

(b) 0.1 wt\% PEG with RB-PEG5k

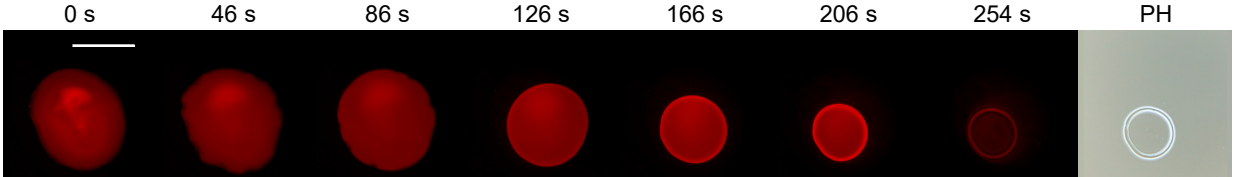

(c)

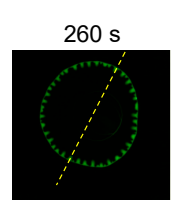

$254 \mathrm{~s}$

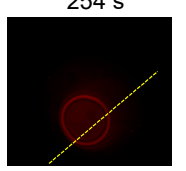

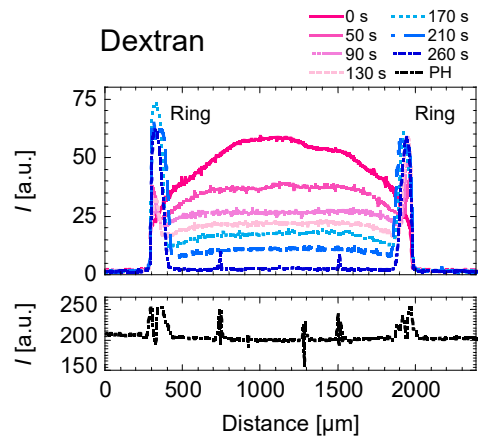

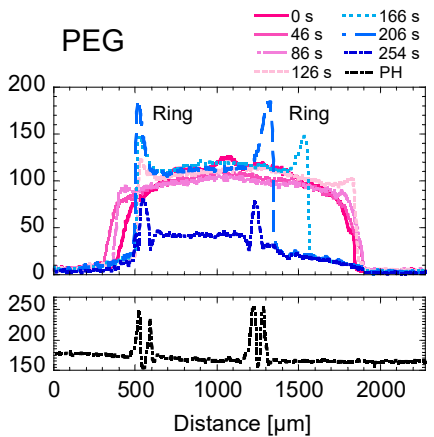

Figure 5. Time evolution of (a) $\mathrm{C}_{\text {tot }}=0.1 \mathrm{wt} \%$ dextran-only and (b) $\mathrm{C}_{\text {tot }}=0.1 \mathrm{wt} \%$ PEG-only droplets upon evaporation. The green and red represent (a) FITC-Dex and (b) RB-PEG, respectively. The phase contrast images on the far right $(\mathrm{PH})$ are taken after $265 \mathrm{~s}$. Scale bar: $1 \mathrm{~mm}$. (c) Intensity line profiles of the dextran or PEG-only droplets at different times (right graphs). The intensity profiles were taken along the yellow dotted lines shown in the fluorescence images (left).

(a)
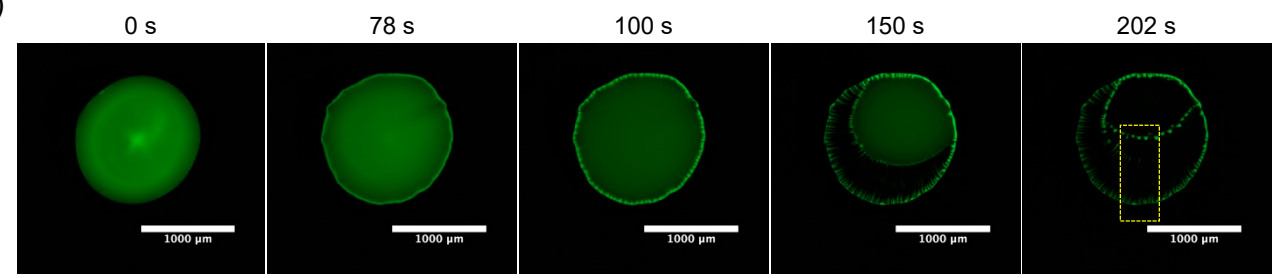

(b)

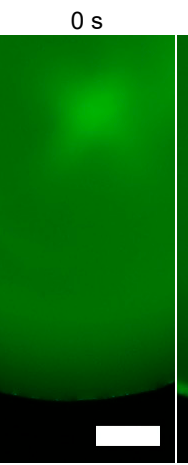

$78 \mathrm{~s}$

$100 \mathrm{~s}$ $124 \mathrm{~s}$ $150 \mathrm{~s}$

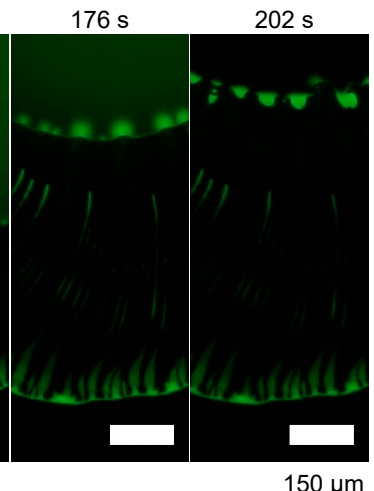

Figure 6. Time evolution of the evaporation patterns for dextran-PEG droplet with $\mathrm{C}_{\text {tot }}=0.1 \mathrm{wt} \%$ and polymer ratio dextran: PEG $=4: 1(w / w)$. Fluorescence images of FITC-Dex (green). (a) Whole droplet images with a scale bar: $1 \mathrm{~mm}$; (b) zoomed views of the part of the droplet surrounded by the yellow square (a). Scale bar: $150 \mu \mathrm{m}$. 

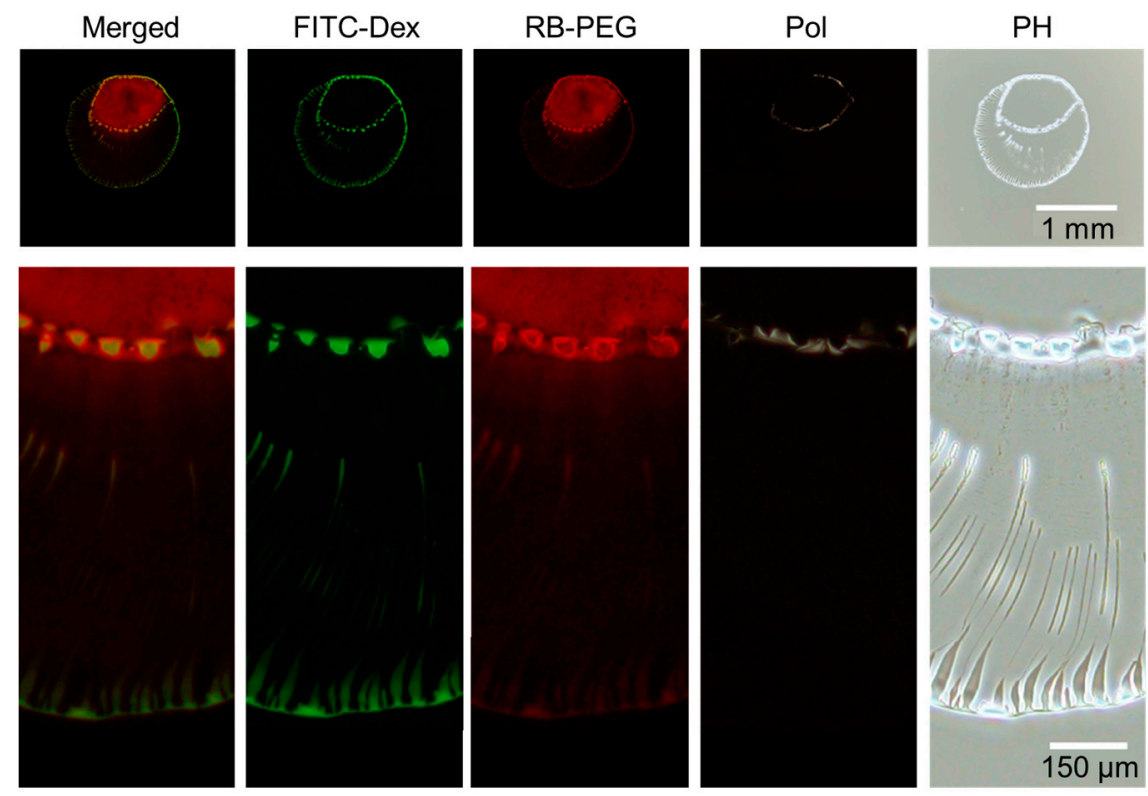

Figure 7. The final deposition images of the droplet shown in Figure 6). From left to right: Merged image of FITC-Dex (green) and RB-PEG (red), FITC-Dex, RB-PEG, simple polarized and phase-contrast microscopy images. Intensity modified for merged and RB-PEG images to obtain better visibility.

After $176 \mathrm{~s}$, the dextran again accumulated to form an inner ring of multiple domains (Figure 6). The visualization of the PEG reveals that the PEG-rich locates inside the inner ring of dextran. The line dividing the inner dextran ring and PEG phase is found to be the phase boundary between dextran and PEG (Figure 7). A closer look at Figure 7 reveals that PEG-rich domains also exist just inside the ring of dextran domains, unlike PEG-only droplets (Figure 3). Therefore, the presence of dextran-rich domains at the phase boundary may contribute to the formation of the PEG-rich domains.

Phase contrast images demonstrate that the inner ring is composed of PEG, unlike the outer ring mainly composed of dextran. In addition, the accumulated PEG ring seems to be bright in polarized image, suggesting that the accumulated PEG ring contains ordered structure, i.e., crystal [17].

\subsection{Initial Contact Angles of Dextran and PEG Droplet}

To understand the pattern $\mathrm{A}$ formation, we measured the initial contact angles $\theta_{\text {int }}$ for dextran-only and PEG-only droplets with some differnet $C_{\text {tot }}$ from 0.01 to $20 \mathrm{wt} \%$ (Figure 8a). Interestingly, dextan and PEG showed the opposite trend of $\theta_{\text {int }}$ against $C_{\text {tot }}$, i.e., the increase in $C_{\text {tot }}$ increases the $\theta_{\text {int }}$ of the dextran droplet (lower wettability with increasing $C_{\text {tot }}$ for dextran) and decreases the $\theta_{\text {int }}$ of PEG (higher wettability with increasing $C_{\text {tot }}$ for PEG), comparable to the water (Figure $8 b$ ).

Under the experimental conditions with $C_{\text {tot }}=0.01$ to $1 \mathrm{wt} \%$ (Figure 3), the dextran and PEG have a differnet $\theta_{\text {int }}$ and a different relationship between $C_{\text {tot }}$ and $\theta_{\text {int }}$. These differences can explain the heterogeneous distribution in the dextran-PEG droplet after 78 $\mathrm{s}$, as shown in Figure 6. The dextran accumulated at the droplet contact line, but the PEG is rather central. At the initial evaporation stage with low $C_{\text {tot }}$, the dextran with smaller $\theta_{\text {int }}$ than the PEG tends to wet the glass substrate. PEG solution easily recedes toward the center, which helps to ensure a stable large $\theta_{\text {int }}$. Upon evaporation, the $\theta_{\text {int }}$ of dextran-rich phase increases. It lowers the contact area of the dextran-rich phase on the substrate and helps to form elongated string-like patterns pulled by the PEG-rich flow. On the contrary, the $\theta_{\text {int }}$ of PEG-rich phase decreases upon evaporation. Initially, the contact line of the PEG solution is moved to maintain the large $\theta_{\text {int }}$; however, at some point, the contact line stops moving and wets the substrate. Since the PEG contact lines do not move, they cannot pull the dextran domains at the phase bundary and change them into strings. In 
fact, Figure 7 demonstrates that the dextran-rich domains at the phase boundary have a spherical shape unlike the elongated shape of dextran connected to the outer ring pattern. From the above, we conclude that the evaporation pattern A (ring pattern with multiple strings) originates from the difference in contact angles between PEG and dextran at a low concentration. In other words, upon evaporation, the dextran-rich phase with a small contact angle accumulates at the contact line of the droplet and forms multiple domains due to density fluctuation, and the PEG phase with large wetting angle deforms it into a string by pulling it. The illustrative time course of the pattern formation is shown in Figure 9.

(a)
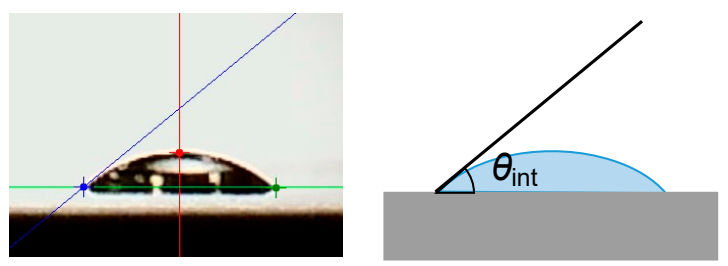

(b)

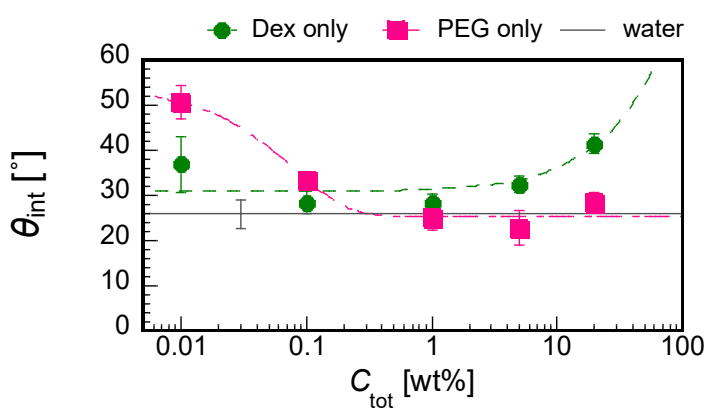

Figure 8. (a) Cross-sectional image of a droplet on a substrate showing how the contact angle $\theta_{\text {int }}$ is measured by the $\theta / 2$ method and the illustration. (b) The initial contact angles for different polymer concentrations for dextran-only (green closed circles) and PEG-only (red closed squares) droplets. The value for water of $25.9 \pm 3.1$ (Ave. $\pm \mathrm{SD}, n=11$ ) is shown with a solid black line with an error bar.

(a)

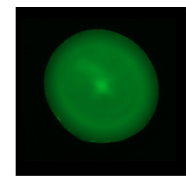

Top

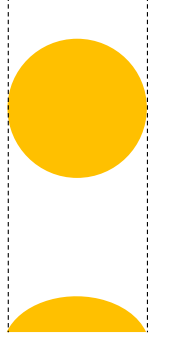

$(\mathbf{b})$

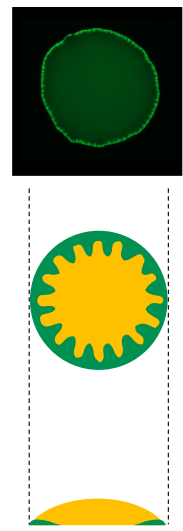

(c)
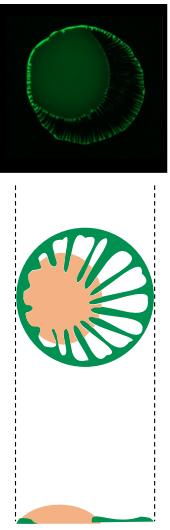

(d)

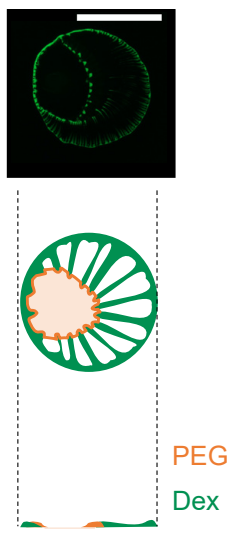

Time

Figure 9. Illustrative time course of evaporation pattern formation. (1) Homogeneous solution just after the droplet deposition, (2) progression of phase separation at the contact line, (3) string formation by the PEG-rich phase receding to the center of the droplet, and (4) complete drying. The microscope images are the same as those shown in Figure 6 (image directions were changed for better visibility). Side views were assumed from the fluorescent intensity profiles. Scale bar: $1 \mathrm{~mm}$. 


\section{Conclusions}

We found a specific evaporation pattern of a dilute dextran-PEG droplets accompanied with phase separation. This pattern consists of multiple strings of dextran pulled from the initial contact line toward the center of the droplet and a concentrated circle of PEG. This pattern was not previously observed for a system using a higher polymer concentration and a lower molecular weight of dextran [13], nor dextran substituted to glucose, which is the monomer unit of dextran. Under our condition, while the macrophase separation occurs at a rather late stage (due to the low initial concentration), the difference in substrate wettability between PEG and long dextran is large, even in the initial stage (as shown in Figure 8). These features may have altered the kinetic coupling between compatibility and wettability, resulting in the characteristic string pattern we found here. This pattern derives from the evaporation mechanism that starts from the very dilute region of the polymer blend, where changes in wettability to the substrate and also differences in compatibility between polymers occur simultaneously. The wettability changes of dextran and PEG to the substrate are opposite, i.e., decreasing for dextran and increasing for PEG. In addition, the decrease in their compatibility with time triggers phase separation. The novel pattern formation found in this study is attributed to the unique kinetic coupling between wettability and compatibility. The study of such complicated evaporation patterns of dilute polymer droplets can be important for diagnosing diseases using a small amount of blood and for considering the primitive environment in which life occurred. The key to understanding the observed phenomenon is the timing of macro-phase separation starts and the time development of the wettability. Therefore, in future work, we would like to analyze the local concentration change and the time evolution of the contact angle to the mixed solution. In addition, the latter can be controlled by the addition of surfactants as tried for the coffee-ring pattern and other studies [18,19]. Moreover, we would like to analyze the viscosity of the solution since it potentially affects the pattern formation.

Author Contributions: Conceptualization, C.W.; investigation, C.W.; resources, C.W. and M.Y.; writing - original draft preparation, C.W.; writing—review and editing, C.W and M.Y.; funding acquisition, C.W. and M.Y. All authors have read and agreed to the published version of the manuscript.

Funding: This research was funded by Japan Society for the Promotion of Science (JSPS) KAKENHI, grant number: 20K14425 (C.W.), 18H01187 (M.Y.) and Japan Science and Technology Agency (JST) ACT-X, grant number: JPMJAX191L (CW).

Institutional Review Board Statement: Not applicable.

Informed Consent Statement: Not applicable.

Data Availability Statement: Not applicable.

Acknowledgments: Our sincere acknowledgement to K. Taguchi (Hiroshima Univ.) for contact angle measurement, S. Tanaka (Hiroshima Univ.) and T. Mouterde (Univ. Tokyo) for insightful discussions.

Conflicts of Interest: The authors declare no conflict of interest.

\section{References}

1. Deegan, R.D.; Bakajin, O.; Dupont, T.F.; Huber, G.; Nagel, S.R.; Witten, T.A. Capillary Flow as the Cause of Ring Stains from Dried Liquid Drops. Nature 1997, 389, 827-829. [CrossRef]

2. Larson, R.G. Twenty Years of Drying Droplets. Nature 2017, 550, 466-467. [CrossRef] [PubMed]

3. Parsa, M.; Harmand, S.; Sefiane, K. Mechanisms of Pattern Formation from Dried Sessile Drops. Adv. Colloid Interface Sci. 2018, 254, 22-47. [CrossRef] [PubMed]

4. Poulard, C.; Damman, P. Control of Spreading and Drying of a Polymer Solution from Marangoni Flows. Europhys. Lett. 2007, 80, 64001. [CrossRef]

5. Chen, R.; Zhang, L.; Zang, D.; Shen, W. Blood Drop Patterns: Formation and Applications. Adv. Colloid Interface Sci. 2016, 231, 1-14. [CrossRef]

6. Pal, A.; Gope, A.; Obayemi, J.D.; Iannacchione, G.S. Concentration-Driven Phase Transition and Self-Assembly in Drying Droplets of Diluting Whole Blood. Sci. Rep. 2020, 10, 18908. [CrossRef] [PubMed] 
7. Sefiane, K.; Duursma, G.; Arif, A. Patterns from Dried Drops as a Characterisation and Healthcare Diagnosis Technique, Potential and Challenges: A Review. Adv. Colloid Interface Sci. 2021, 298, 102546. [CrossRef]

8. Carreón, Y.J.P.; Díaz-Hernández, O.; Escalera Santos, G.J.; Cipriano-Urbano, I.; Solorio-Ordaz, F.J.; González-Gutiérrez, J.; Zenit, R. Texture Analysis of Dried Droplets for the Quality Control of Medicines. Sensors 2021, 21, 4048. [CrossRef]

9. Alberti, S.; Gladfelter, A.; Mittag, T. Considerations and Challenges in Studying Liquid-Liquid Phase Separation and Biomolecular Condensates. Cell 2019, 176, 419-434. [CrossRef] [PubMed]

10. Wegmann, S.; Eftekharzadeh, B.; Tepper, K.; Zoltowska, K.M.; Bennett, R.E.; Dujardin, S.; Laskowski, P.R.; MacKenzie, D.; Kamath, T.; Commins, C.; et al. Tau Protein Liquid-Liquid Phase Separation Can Initiate Tau Aggregation. EMBO J. 2018, 37 , e98049. [CrossRef] [PubMed]

11. Rebane, A.A.; Ziltener, P.; LaMonica, L.C.; Bauer, A.H.; Zheng, H.; López-Montero, I.; Pincet, F.; Rothman, J.E.; Ernst, A.M. Liquid-Liquid Phase Separation of the Golgi Matrix Protein GM130. FEBS Lett. 2020, 594, 1132-1144. [CrossRef] [PubMed]

12. Jia, T.Z.; Chandru, K.; Hongo, Y.; Afrin, R.; Usui, T.; Myojo, K.; Cleaves, H.J. Membraneless Polyester Microdroplets as Primordial Compartments at the Origins of Life. Proc. Natl. Acad. Sci. USA 2019, 116, 15830-15835. [CrossRef] [PubMed]

13. Guo, W.; Kinghorn, A.B.; Zhang, Y.; Li, Q.; Poonam, A.D.; Tanner, J.A.; Shum, H.C. Non-Associative Phase Separation in an Evaporating Droplet as a Model for Prebiotic Compartmentalization. Nat. Commun. 2021, 12, 3194. [CrossRef] [PubMed]

14. Watanabe, C.; Yanagisawa, M. Cell-Size Confinement Effect on Protein Diffusion in Crowded Poly(Ethylene)Glycol Solution. Phys. Chem. Chem. Phys. 2018, 20, 8842-8847. [CrossRef] [PubMed]

15. Harusawa, K.; Watanabe, C.; Kobori, Y.; Tomita, K.; Kitamura, A.; Kinjo, M.; Yanagisawa, M. Membrane Surface Modulates Slow Diffusion in Small Crowded Droplets. Langmuir 2021, 37, 437-444. [CrossRef] [PubMed]

16. Nittmann, J.; Daccord, G.; Stanley, H.E. Fractal Growth Viscous Fingers: Quantitative Characterization of a Fluid Instability Phenomenon. Nature 1985, 314, 141-144. [CrossRef]

17. Biswas, N.; Ichikawa, M.; Datta, A.; Sato, Y.T.; Yanagisawa, M.; Yoshikawa, K. Phase Separation in Crowded Micro-Spheroids: DNA-PEG System. Chem. Phys. Lett. 2012, 539-540, 157-162. [CrossRef]

18. Dewangan, J.K.; Basu, N.; Chowdhury, M. Cationic Surfactant-Directed Structural Control of NaCl Crystals from Evaporating Sessile Droplets. Soft Matter 2022, 18, 62-79. [CrossRef] [PubMed]

19. Inanlu, M.J.; Shojaan, B.; Farhadi, J.; Bazargan, V. Effect of Particle Concentration on Surfactant-Induced Alteration of the Contact Line Deposition in Evaporating Sessile Droplets. Langmuir 2021, 37, 2658-2666. [CrossRef] [PubMed] 